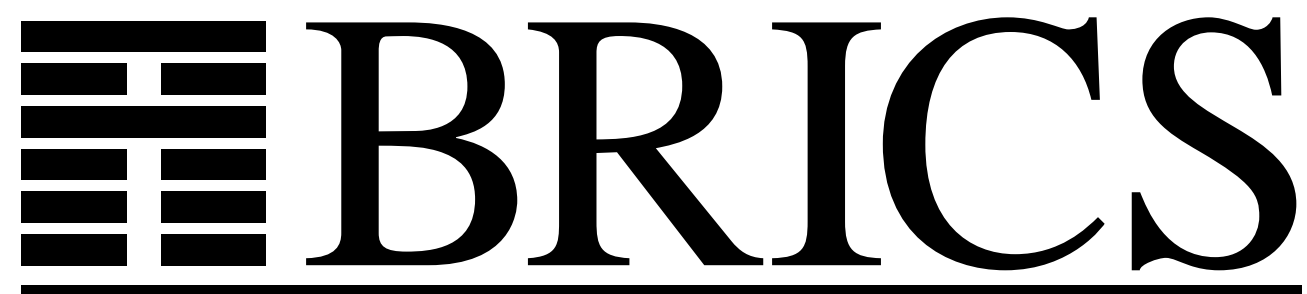

Basic Research in Computer Science

Formal Modeling and Analysis of an Audio/Video Protocol: An Industrial Case Study Using UPPAAL

Klaus Havelund

Arne Skou

Kim G. Larsen

Kristian Lund 
Copyright (c) 1997, $\quad$ BRICS, Department of Computer Science University of Aarhus. All rights reserved.

Reproduction of all or part of this work is permitted for educational or research use on condition that this copyright notice is included in any copy.

See back inner page for a list of recent BRICS Report Series publications. Copies may be obtained by contacting:

\author{
BRICS \\ Department of Computer Science \\ University of Aarhus \\ Ny Munkegade, building 540 \\ DK-8000 Aarhus C \\ Denmark \\ Telephone: +4589423360 \\ Telefax: $\quad+4589423255$ \\ Internet: BRICS@brics.dk
}

BRICS publications are in general accessible through the World Wide Web and anonymous FTP through these URLs:

http://www.brics.dk

ftp: //ftp.brics.dk

This document in subdirectory RS/97/31/ 


\title{
Formal Modeling and Analysis of an Audio/Video Protocol: An Industrial Case Study Using UPPAAL
}

\author{
Klaus Havelund \\ Arne Skou \\ Kim Guldstrand Larsen \\ BRICS*, Aalborg University, Denmark \\ $\{$ havelund,ask,kgl\}@cs.auc.dk \\ Kristian Lund \\ Bang \& Olufsen, Denmark \\ klu@bang-olufsen.dk \\ November, 1997
}

\begin{abstract}
A formal and automatic verification of a real-life protocol is presented. The protocol, about 2800 lines of assembler code, has been used in products from the audio/video company Bang \& Olufsen throughout more than a decade, and its purpose is to control the transmission of messages between audio/video components over a single bus. Such communications may collide, and one essential purpose of the protocol is to detect such collisions. The functioning is highly dependent on real-time considerations. Though the protocol was known to be faulty in that messages were lost occasionally, the protocol was too complicated in order for Bang \& Olufsen to locate the bug using normal testing. However, using the real-time verification tool UPPAAL, an error trace was automatically generated, which caused the detection of "the error" in the implementation. The error was corrected and the correction was automatically proven correct, again using UPPAAL. A future, and more automated, version of the protocol, where this error is fatal, will incorporate the correction. Hence, this work is an elegant demonstration of how model checking has had an impact on practical software development. The effort of modeling this protocol has in addition generated a number of suggestions for enriching the UPPAAL language. Hence, it's also an excellent example of the reverse impact.
\end{abstract}

*Basic Research in Computer Science, Centre of the Danish National Research Foundation. 


\section{Introduction}

Since the basic results by Alur, Courcoubetis and Dill [1,2] on decidability of model checking for real-time systems with dense time, a number of tools for automatic verification of hybrid and real-time systems have emerged [5, 10, 8]. These tools have by now reached a state, where they are mature enough for application on industrial case-studies as we hope to demonstrate in this paper.

One such tool is the real-time verification tool UPPAAL ${ }^{1}$ [5] developed jointly by BRICS at Aalborg University and Department of Computing Systems at Uppsala University. The tool provides support for automatic verification of safety and bounded liveness properties of real-time systems, and it contains a number of additional features including graphical interfaces for designing and simulating system models. The tool has been applied successfully to a number of case-studies $[13,3,4,12,7]$ which can roughly be divided in two classes: real-time controllers and real-time communication protocols.

Industrial developers of embedded systems have been following the above work with great interest, because the real-time aspects of concurrent systems can be extremely difficult to analyse during the design and implementation phase. One such company is Bang \& Olufsen - having development and production of fully integrated home audio/video systems as a main activity.

In 1996, BRICS and Bang \& Olufsen (B\&O) agreed to collaborate on a case study based on one of the company's existing protocols for audio/video device control. The protocol was of interest for three reasons: Firstly, it contained an unexplained error which occasionally caused data loss. The source of this error was unknown to everyone, including $\mathrm{B} \& \mathrm{O}$, prior to the exercise. That is, normal testing had not been sufficient to identify the wrong code. Our goal should be to explain the error. Secondly, the protocol documentation was very low level (consisting solely of assembler listings and flow charts) - so the company could expect an improved documentation as a byproduct of the work. Thirdly, B\&O is about to move (a corrected version of) the protocol to a different platform; thus the case-study will test the benefits of the modeling and verification abilities of UPPAAL in a realistic development process. Finally, the company had no problems in publishing the results in full detail afterwards. Although the protocol is designed for use in audio/video networks, it is a general purpose protocol applicable also in other contexts.

This paper reports the preliminary results of our collaboration. We describe how the UPPAAL tool has been applied in constructing a model of the current protocol implementation. The model was developed via 5 major iteration steps during 3 months, where each new step was motivated by further clarification of the implementation obtained by simulation, trial verification, discussions and code inspection. In the final model, accepted by $\mathrm{B} \& \mathrm{O}$ as valid with respect to the current implementation, we identified a timing error in the collision detection of the protocol implementation (via diagnostic information provided automatically by UPPAAL). Finally, a corrected version

\footnotetext{
${ }^{1}$ See URL: http://www.docs.uu.se/docs/rtmv/uppaal/index.shtml for information about UPPAAL.
} 
of the protocol was suggested and afterwards successfully verified. For each model version, the verification was performed on a suitably reduced model, in order to be manageable by the tool while still allowing the error to be identified.

During the development of models, we found that the notion of timed automata and their graphical representation served extremely well as communication means between the industrial protocol designer and the tool expert doing the simulation and verification. In addition, the graphical simulation features of UPPAAL lead to fast detection of several (obvious) errors in the early models.

The resulting protocol documentation consists of 9 timed automata (a few pages of drawings). This is shorter by an order of magnitude than the original documentation, i.e. a few pages of timed automatons versus 2800 lines of assembler code and 3 pages of flow charts. Most of the original information was immediately available - either via the flowcharts or through discussions. However, a few times we had to walk through the assembler code in order to obtain precise information. The lack of a model (formal or informal) and the fact that the diagnostic trace ${ }^{2}$ of the protocol consisted of close to 2000 transitions-steps, indicates that the error probably would not have been found without the tool assistance. In fact, by using the diagnostic information from the tool, it was possible to provoke the error in B\&O's laboratory. The paper is organized as follows: In sections 2 and 3, we present the UPPAAL tool and the B\&O protocol. In section 4 we present our model of the existing protocol, and in sections 5 and 6 we present the identification of the protocol error and its correction. Section 7 provides concluding remarks, evaluates the UPPAAL tool in retrospective and points out further work.

\section{The UPPAAL model and tool}

UPPAAL is a tool box for symbolic simulation and automatic verification of real-timed systems modeled as networks of timed automata [2] extended with integer variables. More precisely, a model consists of a collection of non-deterministic processes with finite control structure and real-valued clocks communicating through channels and shared integer variables. The tool box is developed in collaboration between BRICS at Aalborg University and Department of Computing Systems at Uppsala University, and has been applied to several case-studies [13, 3, 4, 12, 7].

The current version of UPPAAL is implemented in $\mathrm{C}++$, XFORMS and MOTIF and includes the following main features:

- A graphical interface based on Autograph [6] allowing graphical descriptions of systems.

- A compiler transforming graphical descriptions into a textual programming format.

\footnotetext{
${ }^{2}$ guaranteed by UPPAAL to be the shortest such.
} 
- A simulator, which provides a graphical visualization and recording of the possible dynamic behaviors of a system description. This allows for inexpensive fault detection in the early modeling stages.

- A model checker for automatic verification of safety and bounded-liveness properties by on-the-fly reachability analysis.

- Generation of (shortest) diagnostic traces in case verification of a particular realtime system fails. The diagnostic traces may be graphically visualized using the simulator.

A system description (or model) in UPPAAL consists of a collection of automata modeling the finite control structures of the system. In addition the model uses a finite set of (global) real-valued clocks and integer variables.

Consider the model of figure 1 . The model consists of two components $\mathrm{A}$ and $B$ with control nodes $\{A 0, A 1, A 2, A 3\}$ and $\{B 0, B 1, B 2, B 3\}$ respectively. In addition to these discrete control structures, the model uses two clocks $x$ and $y$, one integer variable $\mathrm{n}$ and a channel a for communication.
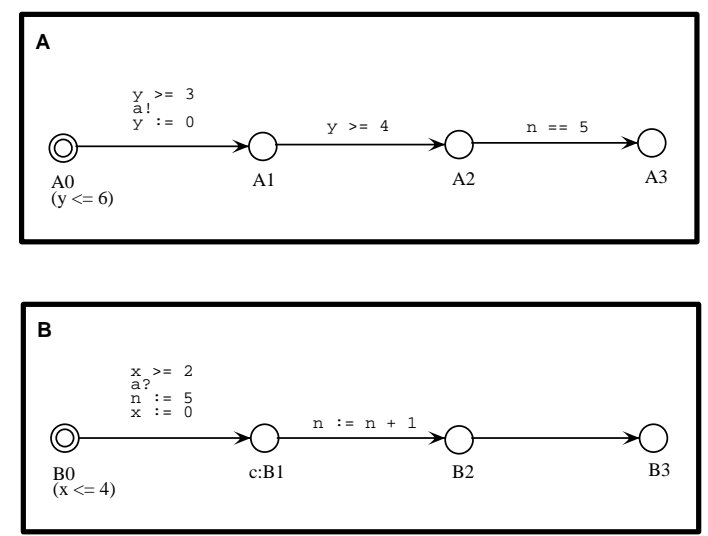

Figure 1: An example UPPAAL model

The edges of the automata are decorated with three types of labels: a guard, expressing a condition on the values of clocks and integer variables that must be satisfied in order for the edge to be taken; a synchronization action which is performed when the edge is taken forcing as in CCS [15] synchronization with another component on a complementary action ${ }^{3}$, and finally a number of clock resets and assignments to integer variables. All three types of labels are optional: absence of a guard is interpreted

\footnotetext{
${ }^{3}$ Given a channel name $a, a !$ and a? denote complementary actions corresponding to sending respectively receiving on the channel a.
} 
as the condition true, and absence of a synchronization action indicates an internal (non-synchronizing) edge similar to $\tau$-transitions in CCS. Reconsider figure 1. Here the edge between $\mathrm{A} 0$ and $\mathrm{A} 1$ can only be taken, when the value of the clock $\mathrm{y}$ is greater than or equal to 3 . When the edge is taken the action $a$ ! is performed thus insisting on synchronization with $\mathrm{B}$ on the complementary action a?; that is for A to take the edge in question, B must simultaneously be able to take the edge from B0 to B1. Finally, when taking the edge, the clock $y$ is reset to 0 . The edge between $A 2$ and A 3 can only be taken if $n$ equals 5 .

In addition, control nodes may be decorated with so-called invariants, which express constraints on the clock values in order for control to remain in a particular node. Thus, in figure 1, control can only remain in $A 0$ as long as the value of $y$ is no more than 6.

Formally, states of a UPPAAL model are of the form $(\bar{l}, v)$, where $\bar{l}$ is a control vector indicating the current control node for each component of the network and $v$ is an assignment giving the current value for each clock and integer variable. The initial state of a UPPAAL model consists of the initial node of all components ${ }^{4}$ and an assignment giving the value 0 for all clocks and integer variables. A UPPAAL model determines the following two types of transitions between states:

Delay transitions As long as none of the invariants of the control nodes in the current state are violated, time may progress without affecting the control node vector and with all clock values incremented with the elapsed duration of time. In figure 1 , from the initial state $\langle(\mathrm{AO}, \mathrm{B} 0), \mathrm{x}=0, \mathrm{y}=0, \mathrm{n}=0\rangle$ time may elapse 3.5 time units leading to the state $\langle(\mathrm{AO}, \mathrm{BO}), \mathrm{x}=3.5, \mathrm{y}=3.5, \mathrm{n}=0\rangle$. However, time cannot elapse 5 time units as this would violate the invariant of $\mathrm{B} 0$.

Action transitions If two complementary labeled edges of two different components are enabled in a state then they can synchronize. Thus in state $\langle(\mathrm{AO}, \mathrm{BO}), \mathrm{x}=$ $3.5, \mathrm{y}=3.5, \mathrm{n}=0\rangle$ the two components can synchronize on a leading to the new state $\langle(\mathrm{A} 1, \mathrm{~B} 1), \mathrm{x}=0, \mathrm{y}=0, \mathrm{n}=5\rangle$ (note that $\mathrm{x}, \mathrm{y}$, and $\mathrm{n}$ have been appropriately updated). If a component has an internal edge enabled, the edge can be taken without any synchronization. Thus in state $\langle(\mathrm{A} 1, \mathrm{~B} 1), \mathrm{x}=0, \mathrm{y}=$ $0, \mathrm{n}=5\rangle$, the $\mathrm{B}-$ component can perform without synchronizing with $\mathrm{A}$, leading to the state $\langle(\mathrm{A} 1, \mathrm{~B} 2, \mathrm{x}=0, \mathrm{y}=0, \mathrm{n}=6\rangle$.

Finally, in order to enable modeling of atomicity of transition-sequences of a particular component (i.e. without time-delay and interleaving of other components) nodes may be marked as committed (indicated by a c-prefix). If one of the components in a state is in a control node labeled as being committed, no delay is allowed to occur and any action transition (synchronizing or not) must involve the particular component (the component is so-to-speak committed to continue). In the state $\langle(\mathrm{A} 1, \mathrm{~B} 1), \mathrm{x}=0, \mathrm{y}=0, \mathrm{n}=5\rangle \mathrm{B} 1$ is committed; thus without any delay the next transition must involve the $\mathrm{B}-$ component. Hence the two first transitions of B are guaran-

\footnotetext{
${ }^{4}$ indicated graphically by a double circled node.
} 
teed to be performed atomically. Besides ensuring atomicity, the notion of committed nodes also helps in significantly reducing the space-consumption during verification.

In this section and indeed in the modeling of the audio/video protocol presented in the following sections, the values of all clocks are assumed to increase with identical speed (perfect clocks). However, UPPAAL also supports analysis of timed automata with varying and drifting time-speed of clocks. This feature was crucial in the modeling and analysis of the Philips Audio-Control protocol [3] using UPPAAL.

\section{Informal protocol description}

In this section we provide an informal presentation of the device control protocol, which is used in existing $\mathrm{B} \& \mathrm{O}$ audio/video equipments. The description is split into protocol environment, protocol syntax, and dynamic protocol rules as advocated in [11].

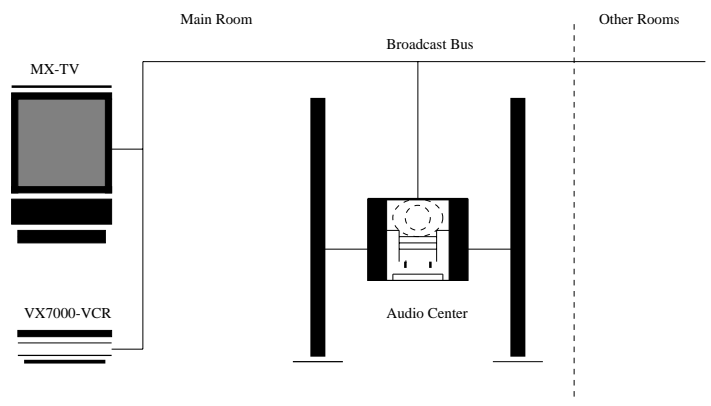

Figure 2: Example B\&O configuration

\subsection{Protocol environment}

The audio/video components in a $\mathrm{B} \& \mathrm{O}$ system are integrated through a broadcast network, called the bus, for command exchange as indicated in figure 2. Examples of commands are start and stop of a VCR initiated via a remote control ${ }^{5}$. Because the bus is shared, there is a risk of collision between component transmissions, and the protocol rules must ensure that collisions are recognized by all involved components in order to prevent data loss or duplication.

\footnotetext{
${ }^{5}$ Typical devices are TV-sets, VCRs, radios, tape recorders, CDs, active loudspeakers etc.
} 


\subsection{Protocol syntax and encoding}

The components exchange information via so-called frames, where each frame consists of a number of T-messages following the abstract syntax:

$$
\text { frame ::= } T_{5}\left\{T_{1}\left|T_{2}\right| T_{3}\right\}{ }^{\geq 15} T_{4}
$$

So, a frame consists of a $T_{5}$, followed by a sequence of at least $15^{6}$ symbols over the set $\left\{T_{1}, T_{2}, T_{3}\right\}$ and terminated by a $T_{4}$. The $T_{i}$ 's have the following roles: $T_{5}$ indicates the start of a frame (used for bus reservation); $T_{4}$ indicates the termination of a frame (used for bus release); and $T_{1}, T_{2}$, and $T_{3}$ are used for the actual frame data. The detailed rules for bus reservation and release are given in section 3.3.

Each T-message $\left(T_{i}\right)$ is represented on the bus as voltage levels ( 0 Volts and 5 Volts) according to the pattern in figure 3 . The figure shows that the $T_{i}$ 's are separated by $0 \mathrm{~V}$ for $1562 \mu s$ - the so-called protocol period. The $T_{i}$ 's are identified by the length of the $5 \mathrm{~V}$ signal between the $0 \mathrm{~V}$ periods. Besides the $T_{i}$ 's, there is an additional pattern called a jamming signal, which is defined as a $0 \mathrm{~V}$ signal for $25 \mathrm{~ms}$.

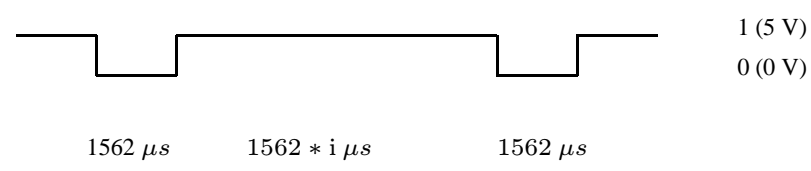

Figure 3: Physical representation of a $T_{i}$ message.

Each component outputs to and reads from the bus via a one-bit register, where 0 represents $0 \mathrm{~V}$, and 1 represents $5 \mathrm{~V}$. When two or more components are accessing the bus, the $0 \mathrm{~V}$ has priority, that is, the bus changes states according to a logical and as described in figure 4 . For the remainder of this paper, we use 0 and 1 to denote both the register values and the voltage levels of the bus.

\begin{tabular}{|c|c|c|}
\hline \hline current bus state & $\begin{array}{c}\text { component output, } \\
\text { new bus state }\end{array}$ & $\begin{array}{c}\text { component output, } \\
\text { new bus state }\end{array}$ \\
\hline $0(0 \mathrm{~V})$ & 0,0 & 1,0 \\
$1(5 \mathrm{~V})$ & 0,0 & 1,1 \\
\hline \hline
\end{tabular}

Figure 4: Rules for changes of bus state

${ }^{6}$ The header size of a frame. A header consists of (format,address,command). 


\subsection{Protocol rules}

Below we describe (in an informal way) the different rules, which must be obeyed when the bus is accessed by a component. We only deal with the sender aspects of a component, as the receiver part is straightforward. Please observe that each component has its own clock - running independently of all other clocks in the system. In order to structure the descriptions, we define the following meta phases for a component: The idle phase, where it waits for a new frame to become ready for transmission, the initialization phase, where it waits for bus reservation, the transmission phase, where the frame transmission takes place, and the collision handling phase, which is entered after a collision detection.

Bus Reservation Rule A network component reserves the bus by issuing a $T_{5}$ and releases the bus by issuing a $T_{4}$ or by detecting a collision and issuing a jamming signal. That is, if a component has issued a $T_{5}$, all other components consider the bus as being reserved.

Frame Gap Rule A network component must ensure the duration of at least $50 \mathrm{~ms}$ between its transmitted frames. However, if a component has generated a jamming signal, it may resend its (destroyed) frame immediately after the jamming signal.

Frame Initialization Rule When a frame becomes ready for transmission (in the idle phase), the sending component delays for $781 \mu \mathrm{s}$ (the reaction delay), enters the initialization phase, and waits for bus reservation. When reservation is possible (i.e. a $T_{5}$ has not been detected on the bus), the component must wait for additional 2 periods and check that the bus state is 1 during the final $781 \mu \mathrm{s}$ of these 2 periods. If this is not the case, bus reservation is retried. Otherwise, another $781 \mu \mathrm{s}$ is awaited, and the transmission phase is entered, starting the transmission of a $T_{5}$.

Bus Sample Rule A sender must sample the bus contents for each period ( $S_{1}$-points in figure 5 ) and in the middle of each period ( $S_{2}$-points in figure 5$)$.

Bus Output Rule A sender must issue output to the bus in the beginning of each sample period (the $W$-points in figure 5). For a given period, the condition $0<\left(W-S_{1}\right)<600 \mu s$ must be satisfied. ${ }^{7}$ In the actual model, we have estimated the quantity $\left(W-S_{1}\right)$ to $40 \mu s$ - the so-called output-delay of the protocol.

Collision Detection Rule A sending component must check the bus for collision at each $S_{2}$-point (see figure 5). For a given period, $s_{1}$ and $s_{2}$ denote the bus values sampled at points $S_{1}$ and $S_{2}$. Furthermore, $p_{n}$ and $p_{f}$ denote the values output to the bus from the component at points $W$ of the given period and its predecessor. A transmission is collision free, if the condition $p_{f}=s_{1} \wedge p_{n}=s_{2}$ is satisfied

\footnotetext{
${ }^{7}$ Due to the physical laws of how fast the bus can change its state.
} 
for each $S_{2}$-point. If this is not the case, the sender enters the collision handling phase.

Collision Handling Rule Due to the priority between voltage levels, a collision can only occur, when 0 is sampled from the bus. Moreover, if the duration of such an (inconsistent) 0 signal is less than 3 periods, the rule is that the component must issue a jamming signal and thereafter reenter the initialization phase. If the duration is at least 3 periods, another component is jamming. The rule is that the sending (non-jamming) component must wait for 18 periods after the 0 signal has disappeared from the bus, and thereafter reenter the initialization phase. This delay gives a jamming component the possibility to retransmit its frame without further collisions.

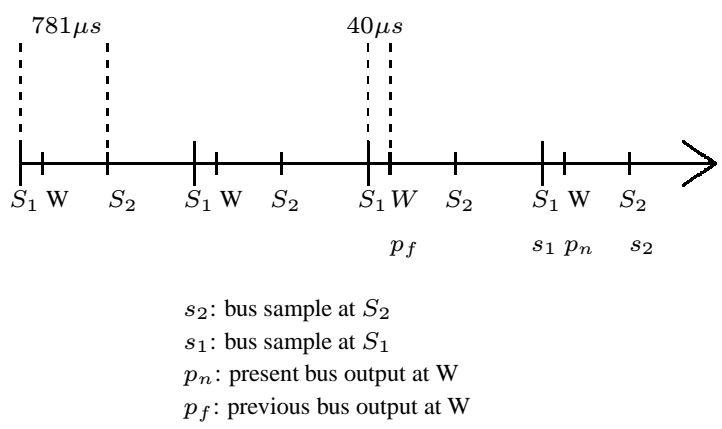

Figure 5: Relative ordering of the variables involved in the collision detection performed at the rightmost $S_{2}$-point

Transmission Stop Rule Whenever a collision has been detected, the component must stop from issuing further bus outputs (and enter the collision handling phase). In this way, it becomes possible to detect if the collision is caused by the jamming of another component.

Detection Stop Rule The final collision detection during frame transmission is the detection performed $781 \mu \mathrm{s}$ after the first 0 signal of the terminating symbol $T_{4}$. Put in another way: When the detection has successfully passed both the period of the leading 0 of $T_{4}$ and also the successor period, the detection must be stopped. This rule avoids 'false' collisions, i.e. collisions, that are detected after the final 0 of a frame.

Protocol Correctness A protocol implementation is correct with respect to collision if the following two conditions are satisfied: (1) if the frame transmitted by a sender $X$ is destroyed (by another sender), then sender $X$ shall detect this; and (2) if one sender detects a collision, then all other simultaneously transmitting senders should detect it. 


\section{A validated formal model of the protocol}

From the informal description given in the previous section it is by no means easy to determine whether the protocol is correct, i.e. satisfies the Protocol Correctness criteria. Thus, in this section we develop a model of the protocol in the UPPAAL language in order to enable a formal automated verification of its correctness using the UPPAAL tool set. We will refer to this model as validated, meaning that it has been approved by $\mathrm{B} \& \mathrm{O}$ as being a correct abstraction of the existing implementation.

The model is - as all models - an abstraction of the real implemented protocol in the sense that it leaves out details regarded as unimportant for the verification task. In our case, an additional challenge in choosing abstraction is the need to reduce the state space to search, and hence to reduce time and space consumption during the automatic verification.

The construction of a model was an iterative process. Several issues had to be right. First of all, the model should be valid, reflecting the code in the protocol, and not do something different. Second, the model should be as abstract as possible to make verification efficient, but detailed enough in order to catch the error, the nature of which we were not aware. Third, the correctness criteria should itself be valid, reflecting a desired property; and fourth, the correctness criteria should be such that the yet unknown error could be caught. The correctness criteria went through a couple of iterations, and was constantly under debate.

We present the complete validated model of the protocol, and from this we shall then derive a reduced model to which the UPPAAL verifier is applied. This reduction is done basically by limiting the number of frames a sender can transmit; and also by limiting the contents of the individual frames: the number of contained T-messages, and their kind. Even with these reductions the protocol will turn out to exhibit erroneous behavior.

\subsection{Overview}

The protocol is modeled in UPPAAL as a network of 9 timed automata (figure 6), which can be divided into three groups: a bus, a sender system named A, and a sender system named B. Note that there are no frame-receivers, as these are not regarded important for the verification task in hand. The sender systems are completely symmetric in their construction, hence, we shall only describe one such, namely system A.

The sender system A consists of four automata: a sender Sender_A, a detector Detector_A, a frame generator Frame_Generator_A and an observer Observer_A. The protocol itself (which is the one implemented in assembler), is here modeled by the sender and the detector. The sender is the key component of the system, and is responsible for transmitting the frames over the bus, while the detector, which is activated from the sender at $\mathrm{S}_{2}$-points, represents the collision detection algorithm.

The frame generator and observer are part of what we will call the environment, hence in principle not components of the implemented protocol. The frame generator basically generates the 0 's and 1's of a frame to be output by the sender, hence it models 


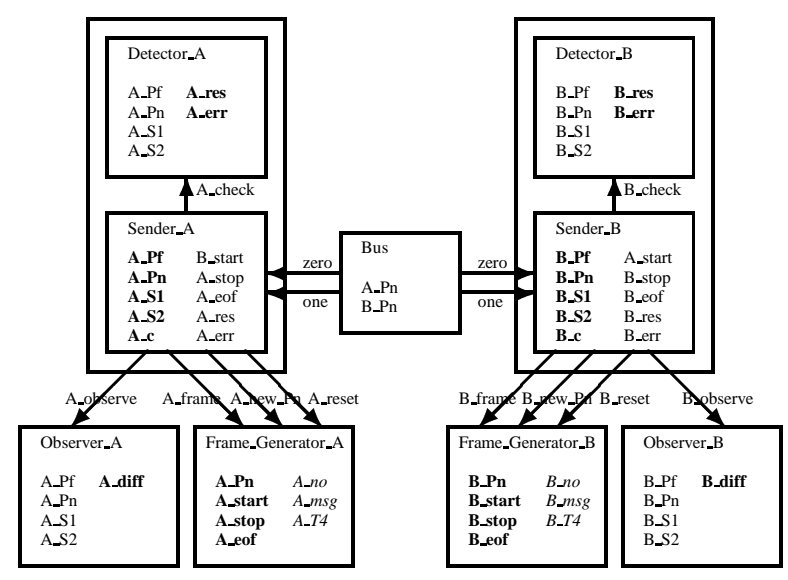

Figure 6: The Protocol

the signals coming for example from a remote control unit. The observer is purely used to formulate the correctness criteria.

The components communicate via channel synchronizations and via shared variables. The figure illustrates the channel connections by arcs going from one component (the one that does a send "!") to another (the one that does a receive "?"). As an example, Sender_A reads the current value of the bus by receiving on either channel zero (value is 0 ) or channel one (value is 1), whichever is enabled. In addition, for each component it is shown (written inside the box) which variables it accesses in which manner. A variable $\mathrm{x}$ is in bold (x) if it is assigned to, and in normal font ( $\mathrm{x})$ if it is only read from. Finally, if a variable is local, it is in italic $(x)$. Note, that by convention a variable may be mentioned in several components if they share it. In a few cases, variables that are only initialized in a component have been omitted for clarity.

\subsection{The bus}

The status of the bus is decided by two variables, $A_{\_} P n$ and $B_{-} P n$, representing the bus registers, as shown in figure 7 . The two variables (initialized to 1 ) are set by the sender systems at W-points by the sending system performing one of the assignments $A_{-} P n$ $:=0$ or A_Pn $:=1$. The senders can sample the actual bus contents by synchronizing on channels zero and one respectively.

\subsection{The frame generator}

The frame generator, figure 8 , is the component that concretely sets the bus by assigning values 0 and 1 to the variable $A_{-} \mathrm{Pn}$ on request from the sender at its $\mathrm{W}$-points. The 


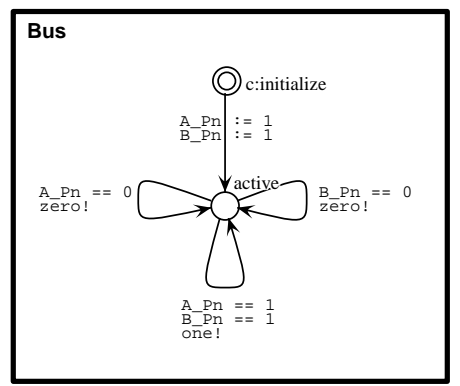

Figure 7: The Bus

generator is initialized by an A_frame! action from the sender, where after each new assignment to A_Pn is triggered by an A_new_Pn! action from the sender, until control returns to the start node. The generator decides what values to assign each time it is triggered by the sender. An A_reset! action from the sender resets the frame generator in case a collision has been detected. Of course one can argue that assigning to A_Pn is not part of the environment; and we could certainly let the generator just produce 0 's and 1's, and let the sender perform the assignments to the bus registers. In fact, such a model existed on our way to the current model, which is however smaller in terms of number of variables used.

Besides A_Pn, three other externally visible variables are assigned to: A_eof, A_stop and A_start. First, the variable A_eof is set to 1 as soon as the last $T_{4}$ message in a frame has been transmitted. The sender will then stop transmitting. Second, according to the Detection Stop Rule, the last collision detection is performed 781 $\mu \mathrm{s}$ after the 0 period beginning the last $T_{4}$ message, and is hereafter disconnected. This is modeled by letting the generator assign the value 1 to the variable A_stop at this point. Finally, according to the Bus Reservation Rule, a precondition for Sender_B to begin transmission of a new frame is that no $T_{5}$ message has been output by Sender_A trying to reserve the bus. Hence, an accurate model would here let Sender_B sample the bus to detect $T_{5}$ 's. This complicates the model unnecessarily, and as an abstraction, we let system A set the variable A_start to 1 when system A has transmitted a $T_{5}$ (to keep the graph simple: at every output of a 0 ending a T-message), and clear it again after the last $T_{4}$, when the bus is released. Sender_B can then read this variable; and vice versa.

Three local variables A_no, A_msg and A_T 4 are used to control the flow of the generator. A frame consists of a sequence of T-messages, which we number from 1 and up. The current T-message number is stored in the variable A_no. The variable A_msg contains the remaining length (in terms of periods) of the current message; that is: the remaining number of 1 's to be output. Recall, that the $T_{5}$ start message consists of 1 's for ten periods (of $1562 \mu \mathrm{s}$ ) or simply ten 1's; hence this variable is initialized to 


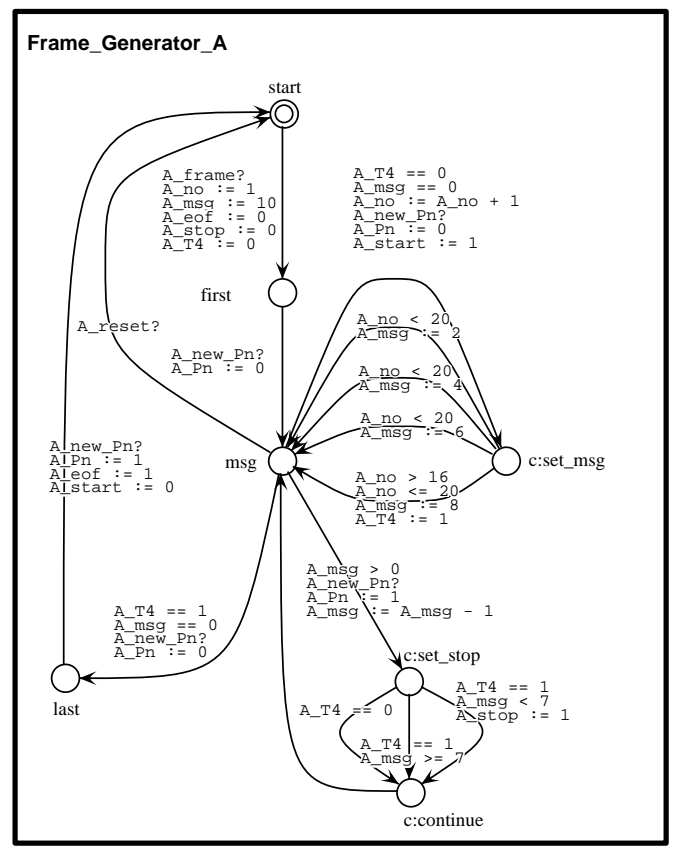

Figure 8: The Generator

10. Finally, the variable A_T 4 is set to 1 when the last $T_{4}$ message is transmitted, just to invoke the exit of the frame generation.

As long as there are messages to transmit, control returns to the msg node. From there the upper right loop is entered each time a 0 is output, and at the same time a non-deterministic choice is made of a new message (length). Note that the lengths of T-messages (in terms of periods, and hence the number of 1's to be output) are as follows: $T_{1}: 2, T_{2}: 4, T_{3}: 6, T_{4}: 8$, and $T_{5}: 10$. The model is limited to transmit minimum 17 and maximum 20 messages (including the starting $T_{5}$ and the ending $T_{4}$ ). This is to limit the search space. The lower right loop is entered for each 1 output to the bus, calculating the value of the A_stop variable each time: when there are less than seven 1's left to be output of the last $T_{4}$ message, collision detection is disconnected.

Note, that the frame generator can be regarded as providing three procedures (the channels), which will be "called" from the sender. The intention is that when the sender "calls" one of these procedures, the sender waits until the "procedure's return". To model such procedure-calls (which are to be performed atomically) in UPPAAL, we have used UPPAAL's committed nodes. This is even more the case for the detector described below. 


\subsection{The detector}

The detector represents the collision detection algorithm, and is to be regarded as a procedure, which, according to the Collision Detection Rule, is "called" from the sender at $\mathrm{S}_{2}$-points, through an A_check! action. As "arguments" it takes the samples $s_{1}$ and $s_{2}$ represented by the global variables A_S 1 and A_S2; and the outputs $p_{f}$ and $p_{n}$ represented by the global variables A_Pf and A_Pn, where after it checks the relationship between these values. The result of the check is written into the variables A_err and A_res. Basically A_err counts the number of 0's sampled, while A_res is set to 0 if no action is to be taken, 1 if sender A should jam, and finally 2 if another sender (B in this case) is jamming. In the latter two cases, sender A should react. The detector is fully made up of committed nodes, hence it consumes no time, and "returns" instantly to the wait_call node after being activated. The graph corresponds closely to a flowchart extracted from the assembler code. The reader is not supposed to grasp the details.

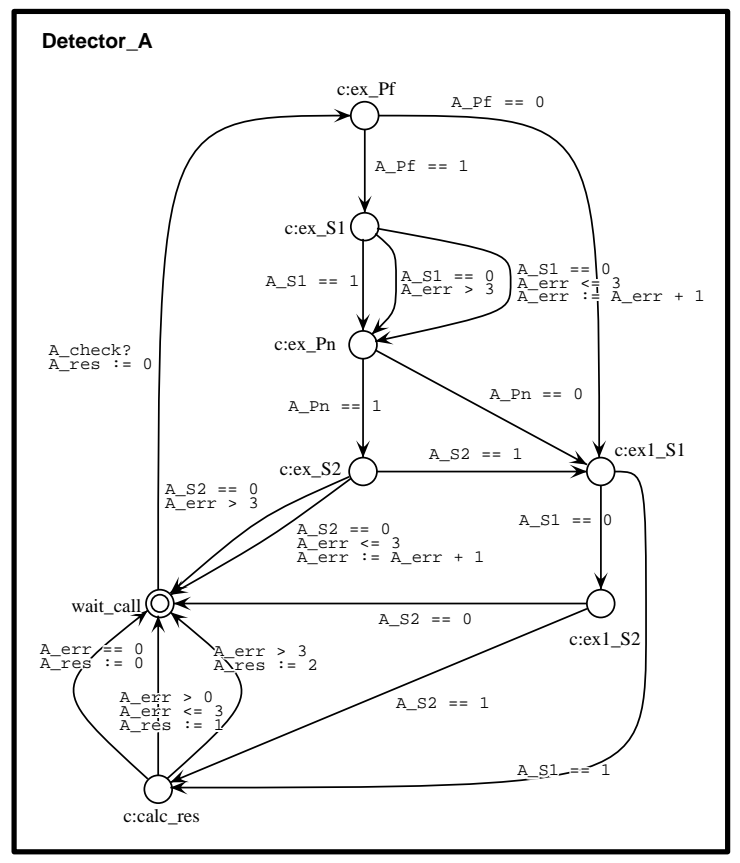

Figure 9: The Detector 


\subsection{The sender}

The sender is responsible for triggering outputs to the bus, and is the main and most complicated component, see figure 10 . It has a single clock, named A_C, which mainly is used to model the timer interrupts that arrive with intervals of $781 \mu \mathrm{s}$. The sendernodes can be divided into three groups: the initialization phase, the transmission phase, and the collision response phase (entered when a collision has been detected, and furthermore a response has been decided).

Initialization phase. This is the upper part of the diagram. The nodes ex_start and other_started model the first part of the Frame Initialization Rule (related to the Bus Reservation Rule), which specifies that no frame can be transmitted if a $T_{5}$ message coming from another sender, B in this case, has been detected on the bus. Recall, that this detection is modeled (abstracted) with the B_start variable being set to 1 by Frame_Generator_B.

The loop at node other_started represents the fact that in case a $T_{5}$ has been detected, then we wait until a $T_{4}$ message is received, releasing the bus. This waiting is done by once every $3124 \mu \mathrm{s}$ to check whether the $T_{4}$ message has been received; here at this abstract level modeled by B_start being equal to 0 again, where after we proceed with the precondition check.

The nodes ex_silencel and ex_silence 2 model the remaining part of the Frame Initialization Rule, where it is specified that the sender must wait further two periods $(2 * 1562 \mu \mathrm{s})$ after the $T_{5}$ reservation check; and in the last $781 \mu \mathrm{s}$ of the second period, the bus must be silent (1). This is modeled by waiting $3 * 781=2343 \mu \mathrm{s}$, and then check the bus value at the beginning and at the end of the remaining $781 \mu \mathrm{s}$ interval. Note how the bus is sampled by synchronizing on either zero? (bus value is 0 ) or one? (bus value is 1 ).

Transmission phase. This is the mid part of the diagram. The transmission starts in node transmit in case the precondition checked in the initialization phase is satisfied. The transition to the check_eof node initializes the frame generator (Frame_Generator_A) via the A_frame! action. The sender now enters a loop, where each iteration represents a period of $2 * 781=1562 \mu \mathrm{s}$. Basically four variables are assigned to during one iteration of this loop: A_Pn in W-points (as we have seen, by Frame_Generator_A), A_P $f$, to hold the previous old value of A_Pn, and finally A_S 1 and A_S 2 to hold the samples in respectively $S_{1}$-points and $S_{2}$-points, as recorded in the Bus Sample Rule.

In the node check_eof it is examined whether an end of frame has been reached, in which case the stop node is entered, and according to the Frame Gap Rule, 50 ms must then pass before a new frame is transmitted. Node check_eof furthermore represents an $\mathrm{S}_{1}$-point where A_S1 is sampled if the frame has not been finished. 


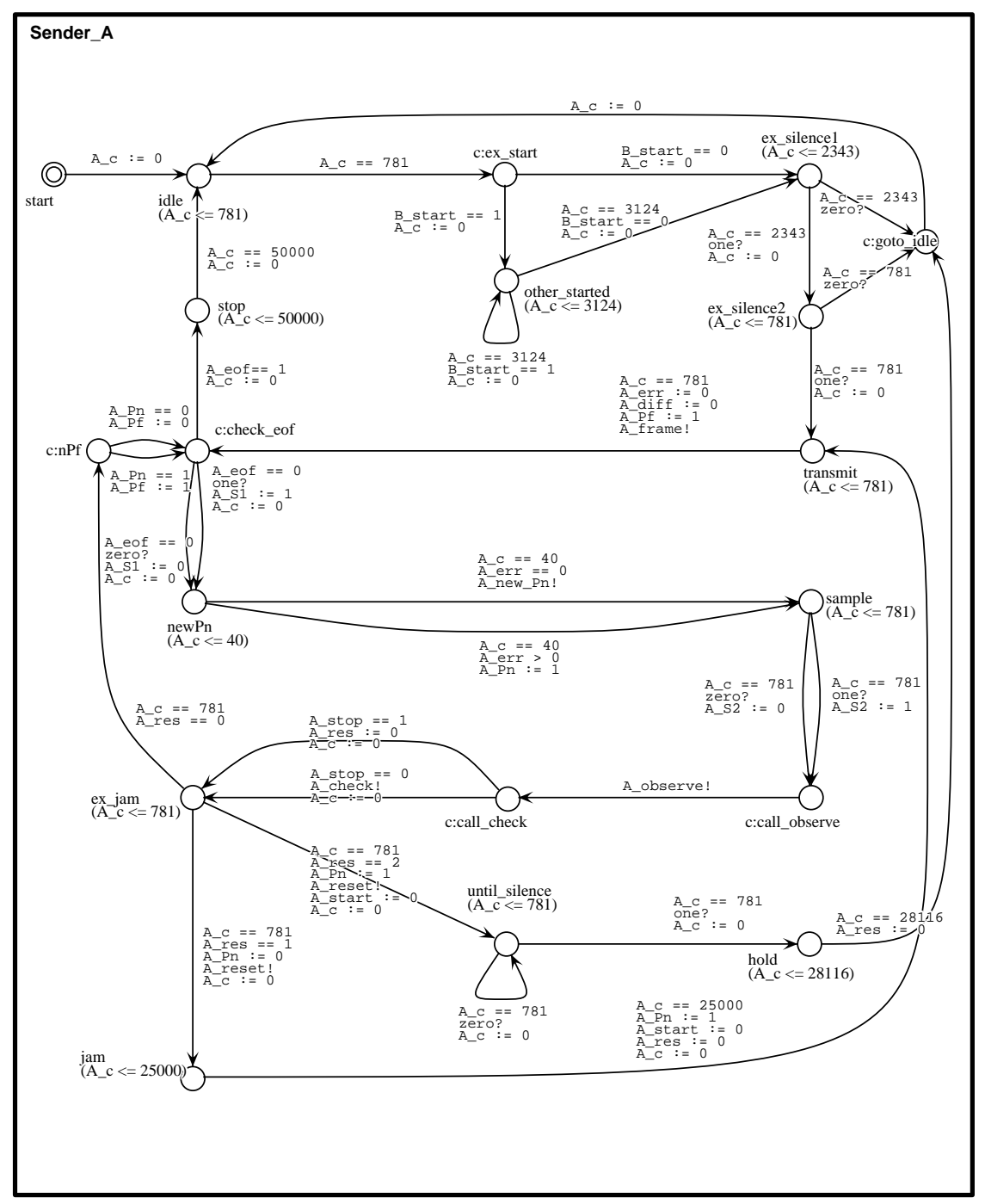

Figure 10: The Sender 
After the sampling, in node newPn, $40 \mu$ s elapses according to the Bus Output Rule before a new value is output, assigned to A_Pn by Frame_Generator_A, which is triggered with the A_new_Pn! action. Note that in case the variable A_err differs from 0 , it means that a collision has been detected, and according to the Transmission stop Rule, transmission should stop, here modeled by just outputting 1's to the bus. In the node sample, A_S2 is sampled, reaching node call_observe. Here the observer and the collision detection, in case not disconnected, are activated. In the ex_jam node the result A_res of the collision detection is examined, and collision response is begun in case it's different from 0 , i.e. either is 1 or 2 , as described in the next paragraph.

Collision response phase. This is the lower part of the diagram. Recall that the value of A_res decides the response. When 1, Sender_A must jam for $25 \mathrm{~ms}$ as stated in the Collision Handling Rule. When 2, another (Sender_B) must be jamming, and we must wait for the bus to be silent, where after 18 periods $(28116 \mu \mathrm{s})$ must pass according to the same rule.

\section{Error detection using UPPAAL}

In this section we shall describe how the error was found in the validated protocol just presented in the previous section. First, the correctness criteria will be formulated, and second, the result of the verification of this criteria, an error trace, will be explained.

\subsection{The correctness criteria}

The correctness criteria is informally stated in the Protocol Correctness statement. It says, that (1) if the frame transmitted by a sender $\mathrm{X}$ is destroyed (by another sender), then sender X shall detect this; and (2) if one sender detects a collision, then every other simultaneously transmitting sender should detect it. In order to formulate rule (1), we must formulate what it means for a frame to be destroyed. We define a frame as destroyed if sampled values differ from output values. Hence, we introduce an observer automaton observing this for each sender, and figure 11 shows the observer for Sender_A.

Recall, that this observer is communicated to from the sender in terms of an A_observe! action at each $\mathrm{S}_{2}$-point. In receiving this signal, the observer sets the variable A_diff to 1 if and only if there is a mismatch between sampled values and output values. That is, if either A_Pf $\neq \mathrm{A}_{-} \mathrm{S} 1$ or A_Pn $\neq \mathrm{A}_{-} \mathrm{S} 2$. This is formulated slightly different in the automaton since UPPAAL does not allow negation in edge guards. Note, that we cannot use Detector_A to observe the relationship between sampled and output values, since this is one of the components we want to verify. With the observer, we are sure to know when output 1's have been destroyed by 0's from another sender. It can easily be shown, that a frame has only been destroyed if at the end of its 


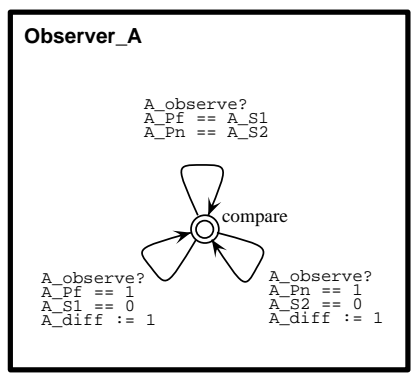

Figure 11: The Observer

transmission A_diff equals 1.

The correctness criteria can now be formulated as follows:

$$
\begin{aligned}
& \text { A[] (A_eof }==1 \text { imply } \\
& \text { (A_diff }=0=0 \text { and B_res }==0) \text { ) }
\end{aligned}
$$

In order to understand this property, note that A_eof is set to 1 when A's frame has been sent, that A_diff is set to 1 if A's frame has been destroyed, and finally, that B_res is set to 1 if $B$ has detected a collision. The property then says, that whenever (A [ ] ) a frame has been sent (A_eof equals 1), the sent frame must be intact (A_diff equals 0 ), and other senders (B in this case) must not have discovered a collision (B_res equals 0). A symmetric property is also verified for sender system B.

\subsection{The error trace}

In order to obtain a fast feed-back (few minutes) during the debugging of the protocol, we worked with a reduced model, where basically each sender only transmitted a single frame of $T_{1}$ messages, surrounded by a $T_{5}$ and a $T_{4}$. This was considered harmless as the purpose was to locate an existing error rather than to prove some property universally true. The verifier rejected the stated correctness criteria as being true, and figure 12 illustrates a condensed version of the error trace produced by UPPAAL ${ }^{8}$ in terms of a bus-value diagram. UPPAAL required 6,27 minutes of computation and $32 \mathrm{M}$ bytes of memory on a Sparc 10.

It appeared to be the Detection Stop Rule that was unhealthy: collision detection seemed to be disconnected too early with the result of messages being lost. The trace describes a scenario, where Sender_A sends a frame of exactly $15 T_{1}$ messages, while Sender_B sends $16 T_{1}$ messages. Hence, the two frames are different, although they are equal up the the last $T_{1}$ of $\mathrm{A}$.

\footnotetext{
${ }^{8}$ The error trace produced by UPPAAL contained 1998 basic transition-steps.
} 


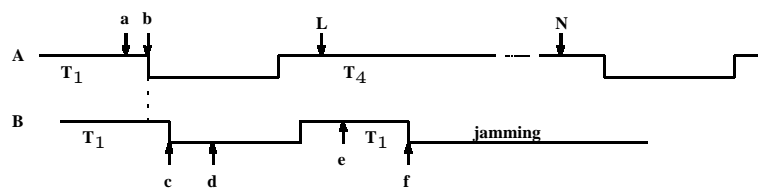

Figure 12: The error trace visualized

Sender_B starts exactly $40 \mu$ s after Sender_A. Precisely this delay, which fatally equals the delay between a senders $S_{1}$-sampling and its bus output, allows the two senders to proceed without any of them discovering their simultaneous bus access. To see this, consider figure 12 which shows how all the 0-periods of the two frames are positioned relative to each other: at point (a) Sender_A samples $S_{1}$ and is ready to output a 0 , but the output happens in point (b) due to the output delay (which is also $40 \mu \mathrm{s}$ ). In point (b) Sender_B now also is ready to sample its $S_{1}$ value. Now, if Sender_A outputs its 0 before B 's sampling, then B will sample a 0 while expecting a 1 , and B will then recognize the collision. However, if Sender_A outputs its 0 after B's sampling, then no collision will be detected by B.

Hence, there is a non-deterministic outcome of each pair of A and B 0-periods: either A will output before B samples, and a collision is detected by B, or A will output after, and no collision will be detected. This mutual ignorance of the collision continues until sender A terminates its last $T_{1}$ message, as illustrated by figure 12, and explained in the following.

The figure in fact illustrates the beginning of the last $T_{4}$ message of Sender_A, together with the beginning of yet another (the 16th) $T_{1}$ message of Sender_B. Up to that point $\mathrm{B}$ has sampled before $\mathrm{A}$ has output and no collision has been detected. Now, however, at point (b), sender A comes first and outputs a 0 , and this is detected by $\mathrm{B}$ in point (d) when the collision detection is activated (B_err $:=B_{-}$err +1 ). In point (e), B then decides to jam (B_res : $=1$ ), which happens in point (f). This is in fact after the last collision detection performed by A in point $(\mathrm{L})$. Hence, sender A never observes the collision, while sender B does. Consequently, A's message is lost and is not retransmitted.

Put differently, and simpler, since a sender disconnects its collision detection early in its $T_{4}$ message, other senders can start jamming after that point without it being detected. The trace violates as well A_diff $==0$ as B_res $==0$ at the point where A_eof $==1$ : sender A's frame is destroyed (without A detecting it), and sender B has detected the collision.

A question is: "how important is it that sender B starts exactly $40 \mu$ s after A?". Well, in the case where both senders send only $T_{1}$ messages, it is important, since if the delay is less than 40 , no collision will ever be detected, and in case the delay is above 40 , collision will be detected immediately by both. This is true in our model. In reality, however, clocks in the various audio/video components may have slightly 
different, and changing, speeds, so in practise senders do not need to start exactly 40 $\mu \mathrm{s}$ apart in order to cause the error.

\section{Correcting the protocol}

Thus, as explained in the previous section, the source of the error was identified as the too early disconnection of the collision detection just after the 0 beginning the last $T_{4}$ message. That is: the last check is performed $781 \mu \mathrm{s}$ after this 0 has been turned back into a 1 , at point (L) in figure 12. This allows another sender to start jamming after this moment without it being detected by the sender having disconnected.

The reason for disconnecting at that early moment is to prevent a frame from being sent twice, since if a collision is detected too late, the frame may in fact have come though, since the collision may not be frame destroying, and a retransmission will then be a duplication. As an example, think of a frame with an information contents like: "go one channel forward". However, it has apparently been disconnected to early, and hence, a solution to the problem is to move the disconnection to a later moment, but not too far since we still want to avoid a frame duplication.

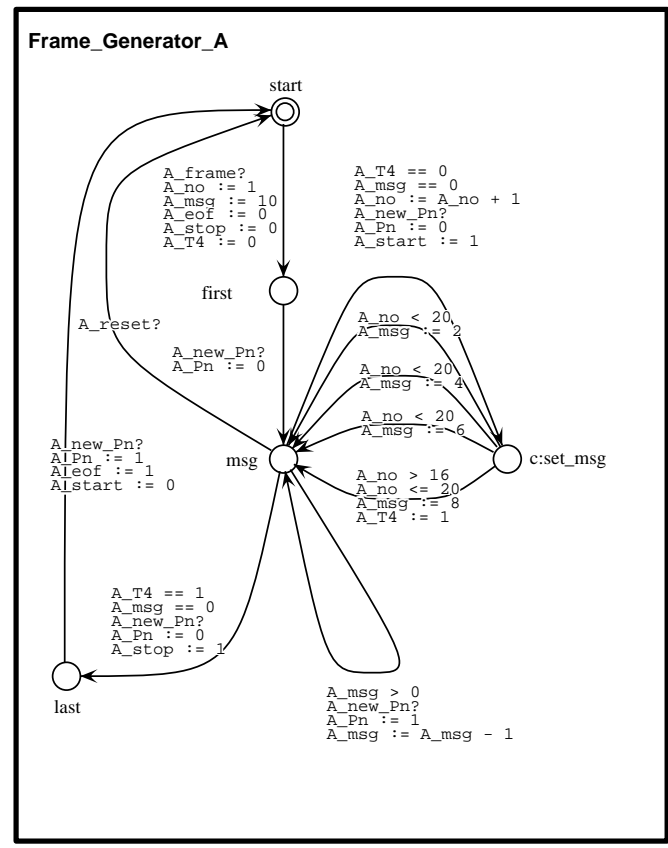

Figure 13: Generator with A_stop: =1 moved 
The solution is to move forward and perform the last collision detection $781 \mu \mathrm{s}$ before the 0 ending the last $T_{4}$ message, at point $(\mathrm{N})$ in figure 12 . Hence, the collision detection is then only disconnected during the last 0 of the whole frame. In our model, this correction must be introduced in the frame generators, and figure 13 shows the new Frame_Generator_A.

The modification consists of moving the assignment A_stop := 1 to a later point, namely to the edge going from node msg to node last. That is, when the last 0 in the last $T_{4}$ message is output. Consequently, the previous assignment to A_stop must be removed resulting in the lower right loop edge leaving and returning to node msg. This edge was before broken into a number of edges over committed nodes, see figure 8 . The observer is also disconnected when the collision detection is (is not shown).

With these modifications, the model was verified correct with respect to the same correctness criteria as presented for the previous model. It required 30 minutes of computation and $90 \mathrm{M}$ bytes of memory on a Sparc 10. The model verified was downscaled to a version where each sender only transmitted one frame, and where sender A only transmitted $T_{1}$ messages (surrounded by a $T_{5}$ and a $T_{4}$ of course), while sender B could transmit the whole range of T-messages.

\section{Conclusions}

The case study clearly showed how model checking can be a help in tracking down undesired behavior in a highly non-deterministic real-time system. The example illustrated the conditions of a real-life problem in the sense that the source of the error (that messages were occasionally lost) was unknown to us, and hence it was not clear at what abstraction level the model should be formulated. This question of abstraction level was also central in the formulation of the correctness criteria. Another kind of abstraction, performed in a second round, consisted of reducing the obtained model to sub-models that could be verified within reasonable time and space. It would be useful to have a workbench which could support easy derivation of verifiable sub-models from a single full model. It turned out, that all sub-models were obtained from the full model by adjusting three different parameters: (1) whether or not a sender transmitted several frames, or just a single frame; (2) how many messages were sent in a single frame; and finally (3) what messages could be transmitted in a single frame.

Choosing the right abstractions were mainly an activity based on intuition, and the adjustment of the parameters mentioned was in addition based on experiments with the model checker. Of course, with such abstractions, one cannot ensure that the protocol in its full complexity is correct even if the model is verified to be. However, such a model can be used to reject the protocol in case errors are found, and this is what happened. Hence, model checking can be seen as a particular advanced kind of debugging where all execution paths in a limited world are examined, rather than some execution paths in a complete world, as in traditional testing. Furthermore, often the abstractions are of such a harmless kind, that even though the correctness of the model does not imply 
the correctness of the protocol, it does increase our confidence in its correctness.

Concerning the error trace, it contained 1998 transition steps, in fact guaranteed to the be shortest trace leading to a state breaking the property to be verified. Examining such a long trace in the simulator turns out to be impracticable, and hence, it was done in an ad hoc fashion (using emacs and its facilities). Research has been initiated to provide means for trace examination, for example by defining a trace simplification language.

Concerning the language for writing atomic edges between nodes, one could consider a Pascal-like programming language, with functions, procedures, control structures like loop and case constructs, and, of course, general datatypes like enumerated types, arrays and records. The Murphi-language [14] - applied to a protocol verification in [9] - could be a good candidate for such a language, and further research will explore this path. As a general comment on the graphical language for writing transition systems it was clearly concluded, that this formalism was ideal in the communication between the tool expert and the protocol designer. The simulator additionally turned out to be of a good help when developing and validating the model before applying the verifier.

\section{References}

[1] R. Alur, C. Courcoubetis, and D. Dill. Model-checking for Real-Time Systems. In Proc. of Logic in Computer Science, pages 414-425. IEEE Computer Society Press, 1990.

[2] R. Alur and D. Dill. Automata for Modelling Real-Time Systems. In Proc. of ICALP'90, volume 443 of Lecture Notes in Computer Science, 1990.

[3] Johan Bengtsson, David Griffioen, Kare Kristoffersen, Kim G. Larsen, Fredrik Larsson, Paul Pettersson, and Wang Yi. Verification of an Audio Protocol with Bus Collision Using UPPAAL. In Proc. of CAV'96, volume 1102 of Lecture Notes in Computer Science. Springer-Verlag, 1996.

[4] Johan Bengtsson, Kim G. Larsen, Fredrik Larsson, Paul Pettersson, and Wang Yi. UPPAAL - A Tool Suite for Symbolic and Compositional Verification of Real-Time Systems. In Proc. of the 1st Workshop on Tools and Algorithms for the Construction and Analysis of Systems, volume 1019 of Lecture Notes in Computer Science. Springer-Verlag, May 1995.

[5] Johan Bengtsson, Kim G. Larsen, Fredrik Larsson, Paul Pettersson, and Wang Yi. UPPAAL in 1995. In Proc. of the 2nd Workshop on Tools and Algorithms for the Construction and Analysis of Systems, number 1055 in Lecture Notes in Computer Science, pages 431-434. Springer-Verlag, March 1996.

[6] A. Bouali, A. Ressouche, and V. Roy R. de Simone. The FC2Toolset. Lecture Notes in Computer Science, 1102, 1996. 
[7] P.R. D’Arenio, J.-P. Katoen, T. Ruys, and J. Tretmans. Modelling and Verifying a Bounded Retransmission Protocol. In Proc. of COST 247, International Workshop on Applied Formal Methods in System Design, 1996.

[8] C. Daws, A. Olivero, and S. Yovine. Verifying ET-LOTOS programs with KRONOS. In Proc. of 7th International Conference on Formal Description Techniques, 1994.

[9] K. Havelund and N. Shankar. Experiments in Theorem Proving and Model Checking for Protocol Verification. In M-C. Gaudel and J. Woodcock, editors, FME'96: Industrial Benefit and Advances in Formal Methods, volume 1051 of Lecture Notes in Computer Science, pages 662-681. Springer-Verlag, 1996.

[10] Pei-Hsin Ho and Howard Wong-Toi. Automated Analysis of an Audio Control Protocol. In Proc. of CAV'95, volume 939 of Lecture Notes in Computer Science. Springer-Verlag, 1995.

[11] Gerard Holzmann. The Design and Validation of Computer Protocols. Prentice Hall, 1991.

[12] H.E. Jensen, K.G. Larsen, and A. Skou. Modelling and Analysis of a Collision Avoidance Protocol Using SPIN and UPPAAL. In The Second Workshop on the SPIN Verification System, volume 32 of DIMACS, Series in Discrete Mathematics and Theoretical Computer Science. American Mathematical Society, 1996.

[13] Magnus Lindahl, Paul Pettersson, and Wang Yi. Formal Design and Analysis of a Gear-Box Controller: an Industrial Case Study using UPPAAL. In preparation., 1997.

[14] R. Melton, D.L. Dill, C. Norris Ip, and U. Stern. Murphi Annotated Reference Manual, Release 3.0. Technical report, Stanford University, Palo Alto, California, USA, July 1996.

[15] R. Milner. Communication and Concurrency. Prentice Hall, Englewood Cliffs, 1989. 


\section{Recent BRICS Report Series Publications}

RS-97-31 Klaus Havelund, Arne Skou, Kim G. Larsen, and Kristian Lund. Formal Modeling and Analysis of an Audio/Video Protocol: An Industrial Case Study Using UPPAAL. November 1997. 23 pp. To appear in The 18th IEEE Real-Time Systems Symposium, RTSS'97 Proceedings.

RS-97-30 Ulrich Kohlenbach. Proof Theory and Computational Analysis. November 1997. 38 pp.

RS-97-29 Luca Aceto, Augusto Burgueño, and Kim G. Larsen. Model Checking via Reachability Testing for Timed Automata. November 1997. 29 pp.

RS-97-28 Ronald Cramer, Ivan B. Damgård, and Ueli Maurer. Span Programs and General Secure Multi-Party Computation. November 1997. 27 pp.

RS-97-27 Ronald Cramer and Ivan B. Damgård. Zero-Knowledge Proofs for Finite Field Arithmetic or: Can Zero-Knowledge be for Free? November 1997. 33 pp.

RS-97-26 Luca Aceto and Anna Ingólfsdóttir. A Characterization of Finitary Bisimulation. October 1997. 9 pp. To appear in Information Processing Letters.

RS-97-25 David A. Mix Barrington, Chi-Jen Lu, Peter Bro Miltersen, and Sven Skyum. Searching Constant Width Mazes Captures the $A C^{0}$ Hierarchy. September 1997. 20 pp. To appear in STACS '98: 15th Annual Symposium on Theoretical Aspects of Computer Science Proceedings, LNCS, 1998.

RS-97-24 Søren B. Lassen. Relational Reasoning about Contexts. September 1997. 45 pp. To appear as a chapter in the book Higher Order Operational Techniques in Semantics, eds. Andrew D. Gordon and Andrew M. Pitts, Cambridge University Press.

RS-97-23 Ulrich Kohlenbach. On the Arithmetical Content of Restricted Forms of Comprehension, Choice and General Uniform Boundedness. August 1997. 35 pp.

RS-97-22 Carsten Butz. Syntax and Semantics of the logic $\mathcal{L}_{\omega \omega}^{\lambda}$. July 1997. 14 pp.

RS-97-21 Steve Awodey and Carsten Butz. Topological Completeness for Higher-Order Logic. July 1997. 19 pp. 